\title{
Dark Matter and Dark Energy as a Derivate from Cosmic Photon Radiation
}

\author{
Guido Zbiral ${ }^{1}$ \\ ${ }^{1}$ Independent Private Scientist, Retired Engineer, Klosterneuburg, Austria \\ Correspondence: Guido Zbiral, Independent Private Scientist, Retired Engineer, Klosterneuburg, Austria. E-mail: \\ guido@zbiral.at
}

Received: November 10, 2015 Accepted: November 23, $2015 \quad$ Online Published: December 28, 2015

doi:10.5539/apr.v8n1p42 URL: http://dx.doi.org/10.5539/apr.v8n1p42

\begin{abstract}
For many decades now, intensive efforts have been undertaken by physicists and cosmologists around the world to investigate dark matter (DM), without noticeable success to date. This situation leads me to believe that one of the assumptions underlying the current doctrine in physics may well be erroneous or incomplete - since a breakthrough in this field of physics and cosmology would otherwise surely have already taken place by now.

For this year (2015) and the following two years, the CERN Nuclear Research Centre has set itself the task of using the LHC (now upgraded to $13 \mathrm{TeV}$ ) to investigate the still completely mysterious phenomenon of dark matter. The researchers at CERN favour the assumption - shared by the majority of physicists and cosmologists - that DM consists of massive non-baryonic particles (so-called WIMPs, Weakly Interacting Massive Particles) hitherto completely unknown to us, which produce a non-baryonic, static gravitational field distributed throughout the entire cosmos.

I cast doubt on the above assumption that DM is massive in nature. As this paper will show, DM can be far better (and more simply) explained in terms of a non-massive gravitational derivate of those photons consumed in the expansion of cosmic space (by performing the work of expansion), those photons thereby being transformed into static physical quantities. This gravitational derivate creates a free gravitational field (decoupled from the other forces of nature) of non-baryonic, static nature, regionally varying in intensity, and this is known as dark matter.
\end{abstract}

Keywords: Baryonic (static) gravitation, non-baryonic dynamic gravitation of photons, non-baryonic static gravitation of dark matter, gravitational quanta; cosmology

\section{Introduction}

As the relativistic mass of photons is of non-baryonic nature, so the gravitation of photons is also non-baryonic. This, in itself, could already indicate a possible relationship between cosmic photons and non-baryonic DM. All further considerations are based both on this and the "Law of Conservation of Gravitation", and ultimately provide a clear and plausible picture concerning the creation and the nature of non-baryonic DM.

Note 1: Law of Conservation of Gravitation: "For each transformation of energy, the quantum of gravitation corresponding to the amount of energy converted in each case is also transferred, so that the effect of the original quantum of gravitation existing before the energy transformation is conserved overall". This conservation principle possesses the character of a hitherto unknown law of nature!

An invaluable (albeit surprising) benefit of this work on DM is that the intractable problem of the mysterious "dark energy" (DE) has been - as it were - unravelled without it being necessary to fall back on Einstein's Cosmological Constant Lambda $(\Lambda)$ or a possible antigravitation or other unconvincing explanations. The surprising result actually obtained is, that DM is so inherently functionally associated with the so-called DE that both of these apparently opposingly acting cosmic phenomena in fact occur as a single dark phenomenon - i.e. only as DM of strongly differing quantity, intensity and locality (refer to 2.13 and 2.14). The obscure DE does not as such exist. 


\section{Physical and Cosmological Assumptions Leading to the Probable Discovery of Dark Matter and to the Unravelling of Dark Energy}

2.01: The current doctrine in physics is that the gravitation of photons is static in nature (any different type of gravitation, e.g. dynamic gravitation, does not hitherto exist for physicists and cosmologists) and can therefore be completely neglected in comparison to the electromagnetic force, which is many orders of magnitude stronger. I disagree with this assumption and I am convinced that the gravitation of photons is just as dynamic in nature as the photons themselves (Zbiral, 2014a, 2014b), because the gravitation is inherently associated with the photons and takes part in their motion. Therefore the gravitation of photons must be produced by gravitational quanta, and thus appears in quantised form. For this reason, the hitherto unknown dynamic gravitation of photons must play a key role in all physical considerations involving photons and electromagnetism.

2.02: With regard to the hypothesis put forward on 2.01, I take the view that each photon consists of two opposingly acting components of quanta, namely an electromagnetic quantum and a corresponding gravitational quantum. The forces associated with both quanta exist in a state of equilibrium with each other precisely at the constant speed of light, i.e. the two opposing dynamic forces are of the same strength! The only way for this equilibrium to be achieved is by the non-baryonic dynamic gravitation of the photons; electromagnetism acting alone (without opposing gravitational force) is unable to produce a stable state of equilibrium. In these circumstances, there is no resulting force acting on the photon - this being a necessary requirement for the constancy of the speed of light.

These two opposingly acting physical quantities of each photon are bound together in an inseparable "dualism". In my opinion, both quanta represent the essence of the photons and of the electromagnetism itself.

2.03: The dynamic gravitation of photons is produced by the photons' gravitational quanta. This is a gravitational radiation of non-baryonic nature inseparably associated with photons, which remains hidden from the external world until the photons are consumed (by performing work, i.e. energy transformation). After this, both quanta are then converted into external static quantities, separated from each other.

2.04: The non-baryonic static gravitational field in the cosmos is generally interpreted by physicists and cosmologists as an effect of dark matter, which only manifests itself in an external form via its gravitational interaction, albeit without revealing the nature of the "matter" causing this gravitation. How dark matter is formed and what it consists of is an issue that physicists and cosmologists have not been able to clarify to date, and indeed some scholars doubt the existence of dark matter. Nevertheless, there is general agreement that non-baryonic static gravitation of unknown origin does indeed exist in the cosmos.

2.05: It is in the nature of energy to be invariably associated with gravitation. Therefore, in analogy with the Law of Conservation of Energy, there must also exist a Law of Conservation of Gravitation, i.e. exactly as is the case with energy, gravitation can neither be created nor annihilated (see note 1).

2.06: Once the cosmic photons performing the work of expansion have exhausted (i.e. transformed) their energy, the gravitational quantum and its non-baryonic dynamic gravitation cannot simply vanish without a trace (or be annihilated), but instead must remain conserved in the transformed form of DM and its non-baryonic static gravitation.

2.07: The photons of the cosmic radiation energy are the permanent driving force for the expansion of cosmic space (Zbiral, 2013b) - this being particularly true of the primordial photons at the beginning of the existence of the cosmos. They perform the work of expansion - which is most energy-intensive in the early supergravitational Planck-Era $\left(10^{-43} \mathrm{sec}\right.$.) of the cosmos, but thereafter dropping off with the continued expansion. The cosmic photons are consumed in this process, i.e., their two dynamic quanta are transformed into static physical quantities. The static transformation product of the consumed electromagnetic energy is the thus expanded cosmic space with its energy content - in compliance with the Law of Conservation of Energy. Since the gravitational quanta, and thus the dynamic gravitation of the photons consumed may also not be lost (Law of Conservation of Gravitation), they too are transformed into static gravitational quantities and deposited in the new space created as a result of the expansion.

With regard to the consistency of the static gravitational quantities it may be stated that, as a derivate of the cosmic photons (CBR, Cosmic Background Radiation) the transformed gravitational quanta cannot be massive in nature; they only serve as pure gravitational quantities, producing a non-baryonic, static gravitational field. 
These gravitational quanta of the cosmic photons consumed in the expansion of the cosmos, which are thereby transformed into a static, physical quantity, represent the long sought-for Dark Matter (Zbiral, 2013a). DM is a purely gravitational form of energy with no massive component that only appears externally through its gravitational interaction.

Note 2: I am convinced, that the non-baryonic DM is of a non-massive nature, being derived from the non-baryonic relativistic mass of cosmic photons. The relativistic mass of photons is itself non-massive in naturein fact it could not possibly be massive due to the fact that photons travel at the speed of light.

2.08: Dark matter is therefore the "static remnant" of cosmic photons (metaphorically speaking a waste product or combustion residue of cosmic photons), which have been and continue to be consumed for the continuing expansion of the universe. DM only manifests itself as a "non-baryonic static gravitative field-energy", distributed in varying densities throughout the entire cosmos.

2.09: DM with its non-baryonic static gravitation is simultaneously deposited in cosmic space where the cosmic radiation energy has actually performed the work of expansion, i.e. in the uniformly expanding cosmic space throughout the universe. The gravitational field energy of DM participates in the expansion of cosmic space (although this is no true for DM in the immediate vicinity of galaxies), being gradually attenuated in the process.

2.10: The so-called DM (which is not in fact a material at all) is a gravitational field energy, whose strength is dependent on the variable energy of the cosmic photons consumed in the course of the cosmic expansion (see (Zbiral, 2013b)); its gravitational force is steadily decreasing in the course of cosmic time and will continue to do so as long as the cosmos goes on expanding.

2.11: Once cosmic photons have performed work (by expanding space), their gravitational quanta and non-baryonic dynamic gravitation are decoupled from the consumed photons and converted into DM with its non-baryonic static gravitation, both of which then lead their own life, independently of the other fundamental forces.

2.12: Whereas non-baryonic DM has been continually created (with an ever lessening energy density) from the very beginning of cosmic creation, baryonic material was only created during the short period of primordial nucleosynthesis, i.e. within the time span of $1 \mathrm{sec}$. to 3 - 5 minutes on the cosmic time scale. This would also explain why there is far more DM than baryonic material in the cosmos.

2.13: Dark matter is continually being created as a result of the continuing expansion of cosmic space. This process was particularly intense at the time of the Big Bang and the subsequent high-energy events in the very early cosmos. The very first cosmic space created within the Planck-Time - and the very first DM created at the same time - represents the very first energy effect of the extremely concentrated cosmic radiation energy. This very first DM created possesses the highest density (intensity) in the entire history of the cosmos; it envelops all subsequent energy effects originating in the course of the continuing expansion of cosmos, thereby forming a boundary to cosmic space, consisting of DM.

2.14: This extremely strong gravitational energy then existing at the boundary of the cosmos has the effect of exercising a powerful attraction on nearby cosmic structures. Seen from our perspective, we perceive an accelerated expansion of the "distant cosmic masses", this phenomenon currently attributed by physicists and cosmologists either to "dark energy" or "antigravitation", producing an accelerated expansion of the "distant cosmic space". In my opinion, however, these are erroneous interpretations.

In specific terms, what we are dealing with here is a "gravitational instability", acting from the boundary of the cosmos with a strong attraction on the baryonic masses located near to this boundary, including the furthermost galaxies detectible today from our perspective.

Note 3: Every spatial area, whose matter or energy density and thus its gravitational effect is somewhat greater than the density of its immediate environment, will gradually become more and more compressed, while adjacent areas of lesser density will become more and more attenuated. This process is called "gravitational instability" and is a requirement for the creation of cosmic structures.

2.15: The cosmos is known to be a "physically closed system". What the physical boundary actually consists of and what conditions prevail there, are questions that natural science (particularly cosmology) has been unable to answer to date. My answer is as follows: The highly concentrated DM in the boundary region of the cosmos is the physical and natural closure of cosmic space. "Beyond" this, "nature" or "physics" as we know it does not exist. 
2.16: The total amount of DM in the cosmos is equivalent to the entire energy of radiation consumed in the expansion of the cosmos from its very beginning until to date, with the greatest amount - exceeding everything else by far - to be found at the boundary of the cosmos.

2.17: This DM, concentrated at the boundary of the cosmos, is far too weak to prevent the further expansion of the cosmos - let alone cause its implosion. This is because the dynamic cosmic background radiation (CBR) continually acts as a driving force exercising a radiation pressure against this elastic boundary, which is stronger than the opposing static gravitational force exerted by the DM collected here. The static force of gravity is by far the weakest of the four forces of nature. To put this into perspective, the electromagnetic force between an electron and a proton acting within an atom is a magnitude of $\mathbf{1 0 ^ { 3 9 }}$ greater than the static force of gravity between the two particles.

2.18: By this means, the two cosmic mysteries of "dark matter" and "dark energy" could now be solved, completely by surprise and also completely at variance to expectations, because:

"Dark energy" is nothing other than a gravitational attraction effect caused by the extremely concentrated dark matter at the boundary of the cosmos acting both on the most distant cosmic structures existing (i.e. beyond our cosmic horizon) and on those very distant structures that are barely perceptible to us! Dark energy as supposed does not exist!

Note 4: In the event that my view of dark matter should turn out to be correct, then the search for dark material in the form of massive non-baryonic particles (so-called WIMPs) in the CERN-LHC cannot be successful - nothing will ever be found. Furthermore, the following question then arises: What type of process is required for non-baryonic material to be created in a head-on collision of two baryonic particles travelling at near-light speed?

\section{Supplementary Information on Gravitation and Dark Matter}

3.1: From today's scientific perspective, only one type of gravitation exists, i.e. static gravitation, while the "dynamic gravitation of photons" is still an unknown phenomenon (or has not yet been noted by the community of physicists and cosmologists). As is well known, static gravitation "decoupled" from the other fundamental forces of nature at a very early stage. This, however, only applies to the by far prevailing non-baryonic static gravitation of DM, the first fundamental force to decouple from the other fundamental forces as early as the Planck Era $\left(10^{-43} \mathrm{sec}\right.$.) and since that point in time has - as it were - led an "independent existence". As DM does not manifestly interact with the other forces of nature, it has remained undetected - and it may even be the case that it will remain undetectable.

3.2: In fact, two types of gravitation exist: The gigantic amount of the unconstrained non-baryonic static gravitation of dark matter (decoupled from the world substrate), and the diminutive amount of static gravitation bound (or coupled) to its baryonic elementary particles! (Other ways to distinguish between them: "static $\leftrightarrow$ dynamic gravitation" as well as "baryonic $\leftrightarrow$ non-baryonic gravitation").

3.3: According to the current perspective of cosmology in relation to the very early formation of the first stars and galaxies, it is only possible to explain the early formation of cosmic structures in terms of the existence of significant quantities of DM even at this very early stage. The formation of the first galaxies solely due to the gravitation of their own baryonic masses would only have ensued much later - if at all - due to the lack of sufficient baryonic gravitation.

3.4: As described above (refer to section 2.13 and 2.14), by far the greatest part of DM was created immediately in the events of the Big Bang and the subsequent quantum era with its greatest density and intensity in the entire cosmic history. Thus it was available to the full extent for the very early formation of cosmic structures. This is a radical insight, shedding new light on the early formation of galaxies.

3.5: Although DM in our perceptible isotropic cosmic space is approximately 5.5 times as large as baryonic matter, the latter only amounts to approx. $5 \%$ of the contents of the cosmos perceivable to us. Nevertheless, this DM only represents an "infinitesimally small residue" in comparison with the DM at the edge of the cosmos. This also explains where the transformation products of the expended radiation energy, originally by far the dominating form of energy in the early radiation dominated era, are now to be found.

This dense yet elastic boundary to the cosmos enables the cosmic background radiation to exist as isotropic black body radiation and to develop an isotropic pressure. This would not be possible without a dense boundary enveloping the cosmos. 
Note 5: During the radiation-dominated era at the onset of cosmic history, the proportion of cosmic radiation energy to the energy possessed by the baryonic material particles was in the order of approx. $10^{10}: 1$. The cosmos was therefore almost entirely a "pure radiation cosmos" containing widely scattered minute "impurities" in the form of baryonic matter, out of which the cosmos we know today subsequently evolved. According to the latest insights in this matter, the radiation-dominated era only lasted for a very short time (approx. 3 to 5 minutes) - up to the end of the period of primordial nuclear synthesis. Then began the material-dominated era.

The almost total consumption of the cosmic radiation energy, far overriding everything else, that took place during the brief radiation-dominated era must have produced vast amounts of transformation products (see 2.13 and 2.14), which collected at the boundary of the cosmos - and thus lies far beyond our cosmic horizon.

3.6: This boundary or "physical closure of the cosmos" consists exclusively of the most concentrated DM ever created in cosmic history. On a large scale, the homogeneity and isotropy of the cosmos applies everywhere in the cosmos - except at its boundary. Here a discontinuity exists with respect to the entire "inner cosmos" as a direct consequence of the discontinuity existing at the moment of the Big Bang. Therefore, the boundary of the cosmos represents "today's invisible image of the Big Bang".

Note 6: Why should it be, though, that of all the events that have taken place in the course of cosmic creation, only those most powerful and striking processes involved at the time zero and directly afterward should have left no "permanent impressions" in the boundary region of cosmic space, while, in contrast, all subsequent cosmic events have indeed left their mark in the corresponding depth of cosmic space? I am certain that the Big Bang too has left its mark in the maximum depth of cosmic space, but its mark remains invisible for ever.

\section{Conclusions}

4.1: When the cosmic photons have performed their work of cosmic expansion, their gravitational quanta are transformed into static gravitational quantities - and this is what is known as "dark matter".

4.2: Dark matter is a non-massive gravitational derivate of consumed cosmic photons, deposited in the simultaneously expanding cosmos and creating a non-baryonic static gravitational field.

4.3: The so-called "dark energy" is in fact a "gravitational instability" between the enormously concentrated DM at the boundary of the cosmos and the remotest galaxies. "Dark energy" per se does not exist.

\section{Acknowledgements}

My warmest thanks go to my translator C. A. Szwaja (M.A. Physics, Oxon), both for translating my manuscript from German into English and his numerous valuable suggestions on the text itself.

\section{References:}

Zbiral, G. (2013a). Innovative Thoughts on the Nature of "Dark Matter" and "Dark Energy". International Journal of Theoretical and Applied Physics (IJTAP), 3(I), 1-14.

Zbiral, G. (2013b). Cosmic Photon Radiation as the Driving Force for the Expansion of the Universe in Accordance with Hubble's Law. International Journal of Theoretical and Applied Physics (IJTAP), 3(I), 25-35.

Zbiral, G. (2014a). The "Dynamic Gravitation of Photons: A Hitherto Unknown Physical Quantity". New Aspects on the Physics of Photons. Journal of Modern Physics, 5, 198-204. http://dx.doi.org/10.4236/jmp. 2014.55030

Zbiral, G. (2014b). The Dynamic Gravitation of Photons from the Perspective of Maxwell's Wave Equations. Journal of Modern Physics, 5, 1094-1096. http://dx.doi.org/10.4236/jmp.2014.512111.

\section{Copyrights}

Copyright for this article is retained by the author(s), with first publication rights granted to the journal.

This is an open-access article distributed under the terms and conditions of the Creative Commons Attribution license (http://creativecommons.org/licenses/by/3.0/). 